\title{
Single-Port Cholecystectomy and Quality of Life: Reply
}

\author{
Markus J. Wagner
}

Published online: 17 October 2013

(C) Société Internationale de Chirurgie 2013

To the Editor,

We thank Mr. Cotton for his interest in our studies and constructive questions on this topic [1]. To our surprise, one of the main results of our study was that the quality of life (QoL) in the single-port group (SPA) was rated higher than that for standard laparoscopic cholecystectomy (LC), even at 6 months postoperatively. This issue achieved significance in the dimensions of mobility, self-care and anxiety.

As mentioned in our discussion, studies that have been concerned with QoL previously collected data mostly during hospitalization and up to a period of 4 weeks postoperatively. They also reported different outcomes. Thus, we were interested in whether the surgical technique had an impact on Qol over a longer time frame.

We defined the baseline for the QoL score survey as 6 months after surgery. Among our initial 222 study patients, 190 were eligible for evaluation at this point in time. Of these 190 patients, 131 (69\%) completed the EQ-5D survey. In contrast to other studies, we did not use a "singlesurgeon survey" because it does not reflect everyday hospital life and the reality of care, at least in Germany. Instead, we tried to classify the expertise of the surgeon and to analyze its influence on the patients outcome.

The intervention was performed by highly experienced surgeons in $98 \%$ of the patients in the SPA group, whereas a heterogeneous group of 11 surgeons of different training levels performed the surgery in the LC group. This corresponds to the reality in a teaching hospital as cholecystectomy is used as a training experience for new surgeons. Hence, the expertise of the surgeons we have divided into three categories and defined as an influencing variable (see Table 1 in the article) and accounted for by multivariate regression analysis. Table 5 makes it clear which factors significantly contributed to the operation time.

We demonstrated that in comparable patient-related findings the procedure was $23 \mathrm{~min}$ faster when performed by an experienced surgeon, than when done by a resident. As we showed in detail in our discussion and summary, this means a lower operating room load. Also, (along with the ports system) of course it is a cost factor, which may be compensated by the shorter hospital stay (median 1 day shorter). To what extent cosmesis and better QoL are worthwhile factors remains unanswered.

\section{References}

1. Cotton M (2013) Single-port cholecystectomy and quality of life. World J Surg. doi:10.1007/s00268-013-2170-y 